\title{
Intrinsic Aerobic Capacity Affects Hippocampal pAkt and HSP72 Response to an Acute High Fat Diet and Heat Treatment in Rats
}

Li Gan ${ }^{\mathrm{a}, \mathrm{b}}$, Xiaonan Wan ${ }^{\mathrm{c}}$, Delin Ma ${ }^{\mathrm{b}, \mathrm{d}}$, Fu-Chen Yang ${ }^{\mathrm{b}}$, Jingpeng Zhu ${ }^{\mathrm{c}}$, Robert S. Rogers ${ }^{\mathrm{b}}$, Joshua L. Wheatley ${ }^{\mathrm{b}}$, Lauren G. Koch ${ }^{\mathrm{e}}$, Steven L. Britton ${ }^{\mathrm{f}}$, John P. Thyfault ${ }^{\mathrm{b}, \mathrm{g}}$, Paige C. Geiger ${ }^{\mathrm{b}}$ and John A. Stanford ${ }^{\mathrm{b}, \mathrm{h}, *}$

${ }^{a}$ Department of Neurology, The Second Affiliated Hospital of Nanchang University, Nanchang, Jiangxi, China

${ }^{\mathrm{b}}$ Department of Molecular and Integrative Physiology, University of Kansas Medical Center, Kansas City, KS, USA

${ }^{\mathrm{c}}$ The Second Clinical Medical College of Nanchang University, Nanchang, Jiangxi, China

${ }^{\mathrm{d}}$ Department of Endocrinology, Tongji Hospital, Tongji Medical College, Huazhong University of Science and Technology, Wuhan, Hubei, China

${ }^{\mathrm{e}}$ Department of Physiology and Pharmacology, The University of Toledo, Toledo, OH, USA

${ }_{\mathrm{f}}^{\mathrm{f}}$ Department of Anesthesiology, University of Michigan, Ann Arbor, MI, USA

${ }^{\mathrm{g}}$ Research Service, Kansas City VA Medical Center, Kansas City, MO, USA

${ }^{\mathrm{h}}$ Kansas Intellectual and Developmental Disabilities Research Center, University of Kansas Medical Center, Kansas City, KS, USA

Accepted 3 May 2021

Pre-press 26 May 2021

\begin{abstract}
.
Background: Aerobic capacity is associated with metabolic, cardiovascular, and neurological health. Low-capacity runner (LCR) rats display low aerobic capacity, metabolic dysfuction, and spatial memory deficits. A heat treatment (HT) can improve metabolic dysfunction in LCR peripheral organs after high fat diet (HFD). Little is known about metabolic changes in the brains of these rats following HT.

Objective: Our objective was to examine the extent to which high or low aerobic capacity impacts Akt (a protein marker of metabolism) and heat shock protein 72 (HSP72, a marker of heat shock response) after HFD and HT in hippocampus.

Methods: We measured phosphorylated Akt (pAkt) in the striatum and hippocampus, and HSP72 in the hippocampus, of HFD-fed and chow-fed LCR and high-capacity runner (HCR) rats with and without HT.

Results: pAkt was lower in the hippocampus of chow-fed LCR than HCR rats. HFD resulted in greater pAkt in LCR but not HCR rats, but HT resulted in lower pAkt in the LCR HFD group. HSP72 was greater in both HCR and LCR rat hippocampus after HT. The HFD blunted this effect in LCR compared to HCR hippocampus.
\end{abstract}

\footnotetext{
${ }^{*}$ Correspondence to: John A. Stanford and Li Gan, Department of Molecular and Integrative Physiology, University of
}

Kansas Medical Center, Kansas City, KS 66160, USA. E-mails: jstanford@kumc.edu and lgan0316@outlook.com 
Conclusion: The abnormal phosphorylation of Akt and diminished HSP response in the hippocampus of young adult LCR rats might indicate early vulnerability to metabolic challenges in this key brain region associated with learning and memory.

Keywords: Acute high fat diet, Akt, heat treatment, intrinsic aerobic running capacity

\section{INTRODUCTION}

Low aerobic capacity is strongly associated with development of type 2 diabetes, obesity, cardiovascular disease, metabolic syndrome, aging, and all-cause mortality [1-5]. Low-capacity runner (LCR) rats show markers of decreased mitochondrial function in skeletal muscle, heart, and liver [6-8]. We previously reported that, compared to high-capacity runner (HCR) rats, mitochondrial markers (e.g., PGC1- $\alpha$, Nrf-1, and TFAM proteins) were lower in young LCR striatum but not in hippocampus [9]. Meanwhile, convincing epidemiological, clinical, and genetic studies provid substantial evidence supporting a link between type 2 diabetes and Alzheimer's disease (AD) [10, 11]. Both young adult and aged LCR rats exhibit spatial memory deficits [12-15]. In aged LCR rats, these cognitive deficits are accompanied by hyperphosphorylated tau accumulation and neuron loss. These findings support the LCR rats as a model linking impaired metabolic function and $\mathrm{AD}$.

Previous research reported that peripheral organs like the liver and skeletal muscle (and the striatum in the brain) are responsive to the metabolic challenge of a 3-day high fat diet (HFD) [9, 16, 17]. Meanwhile, Geiger et al. reported that heat treatment (HT) improves metabolic dysfunction in liver and skeletal muscles of LCR rats following a 3-day HFD [16]. The goal of the current study was to determine the extent to which a 3-day HFD and HT affect protein markers of metabolism and the heat shock response in LCR hippocampus, as they did in metabolicallysensitive peripheral organs. We used tissue harvested from rats that were used in our recently-published study that focused only on the effects of a HFD in LCR and HCR rats $[9,16,17]$.

\section{MATERIALS AND METHODS}

\section{Animals, diets, and HT}

Seven-month-old male LCR and HCR rats $(\mathrm{N}=48$, generation 25) in our study were the same rats used in our recently-published study [9]. All procedures were approved by the Institutional Animal Care and Use Committees at the University of Kansas
Medical Center, University of Michigan, and University of Missouri, as well as the Subcommittee for Animal Safety at the Harry S. Truman Memorial VA Hospital. Animals were housed in a temperature controlled facility with $12: 12 \mathrm{~h}$ light: dark cycles. The rats were provided standard chow diets (D12450B, 10\% kcal from fat; Research Diets, New Brunswick, NJ) and water ad libitum prior to initiation of the experimental diet period. Rats were placed on either the chow diet or a HFD (D12451, 45\% of kilocalories from fat; Research Diets) for 3 days.

After feeding for 3 days, rats were anesthetized with ketamine-xylazine $(80 \mathrm{mg} / \mathrm{kg}$ body wt $-10 \mathrm{mg} / \mathrm{kg}$ body wt) and received a single heat or sham treatment in vivo. As a result, four treatment groups with HCR/LCR strains were generated: Chow-Sham, HFD-Sham, Chow-Heat, and HFD-Heat. HT consisted of lower body immersion in a $42^{\circ} \mathrm{C}$ water bath to gradually raise body temperature to between $41^{\circ} \mathrm{C}$ and $41.5^{\circ} \mathrm{C}$ where it was maintained for $20 \mathrm{~min}$. Temperature was monitored by a rectal thermometer. Sham treatment consisted of immersion in a $37^{\circ} \mathrm{C}$ water bath and maintaining body temperature.

\section{Tissue harvest}

Following a $10 \mathrm{~h}$ overnight fast, animals were deeply anesthetized with pentobarbital sodium $(50 \mathrm{mg} /$ $\mathrm{kg}$ ). Brains were then removed and immediately placed on an ice cold brain block. Samples of hippocampus and striatum were carefully dissected, weighed, and frozen on dry ice, and stored into $-80^{\circ} \mathrm{C}$ for western blotting analysis.

\section{Western immunoblotting}

Hippocampus and striatum tissue were processed for western blotting by methods previously described [9]. Films were scanned at high resolution, and densitometry measurements were analyzed using Image J software. Protein content was normalized to the loading control $\beta$-actin. Primary antibodies used included phospho-Akt (Ser473) (Cat \# 9271, Cell Signaling), total Akt (Cat \# 9272, Cell Signaling) and HSP72 (Cat \# SPA-810, Enzo Life Sciences, Farmingdale, NY). Secondary antibodies used included goat anti-rabbit 
(Cat \# sc-2004, Santa Cruz) and goat anti-mouse (Cat \# 170-5047, BioRad, Hercules, CA).

\section{Statistical analyses}

All data were presented as means \pm SEM and was analyzed using SPSS. Results were analyzed using a one-way ANOVA followed by post hoc test (Fisher's LSD). Partial eta squared $\left(\eta_{\mathrm{p}}{ }^{2}\right)$ values indicate effect sizes and $p$ values are unprotected for multiple comparisons (a limitation). Significance was set at $p<0.05$.

\section{RESULTS}

Region-specific difference in pAkt/Akt expression and its response to a 3-day HFD in HCR and LCR rat brain

In the hippocampus, pAkt/Akt differed significantly between the four groups $\left(p=0.036, \eta_{\mathrm{p}}{ }^{2}\right.$ $=0.26$ ). pAkt/Akt was lower in chow-fed LCR than chow-fed HCR rats ( $p=0.039$, Fig. 1A). pAkt/Akt was greater in the hippocampus of HFD LCR group than in the chow-fed LCR group ( $p=0.007$, Fig. 1A). There were no significant LCR-HCR differences in pAkt/Akt levels in the striatum, and a 3-day HFD did not affect pAkt/Akt in this brain region $(p>0.05$, Fig. 1B).

\section{HT affected pAkt/Akt with different diets in HCR and LCR hippocampus}

The effects of HT on pAkt/Akt in HCR hippocampus did not differ significantly between the two diet groups as shown in Fig. 1C. In the LCR rats, HT did affect pAkt/Akt differently across the diet groups $\left(p=0.016 ; \eta_{\mathrm{p}}{ }^{2}=0.29\right)$. In chow diet groups, HT did not affect pAkt/Akt in LCR hippocampus. After a 3-day HFD, pAkt/Akt of LCR hippocampus was lower in the HT groups than sham groups $(p=0.042$, Fig. 1D).

\section{Heat shock response in HCR and LCR hippocampus with different diets}

In the HCR rats, HT resulted in a significant overall effect between-groups ( $p=0.019 ; \eta_{\mathrm{p}}{ }^{2}=0.30$ ). HT resulted in greater HSP72 levels in HCR rat hippocampus $(p=0.01$, Fig. 2A). This effect was not altered by a 3-day HFD ( $p>0.05$, Fig. 3A). In the LCR rats, the HT resulted in a significant overall effect between-groups ( $\left.p=0.004 ; \eta_{\mathrm{p}}{ }^{2}=0.34\right)$. HT resulted in greater HSP72 protein expression ( $p=0.003$, Fig. 2B) in chow-fed LCR hippocampus. Unlike HCR rats, HSP72 levels were lower in the HFD versus chow LCR rats following HT ( $p=0.025$, Fig. 2B).

\section{DISCUSSION}

In this study, we report lower pAkt in the hippocampus of LCR rats compared to HCR rats. pAkt did not differ between strains or diets in striatum. However, LCR hippocampus exhibited pAkt levels that were similar to those in HCR rats after a 3day HFD. Hippocampal pAkt did not differ between chow-fed and HFD-fed HCR rats. HT resulted in lower pAkt levels in LCR HFD rats. Our results show HSP72 is elevated in both HCR and LCR rat hippocampus after HT. However, HFD blunted the induction of HSP72 by HT in LCR rat hippocampus compared to the HCR group.

Akt (protein kinases B, PKB) is a critical regulator of cell growth, survival, proliferation, and metabolism. pAkt is Akt activated by phosphorylation at Ser473. Activation of Akt is particularly important for protecting neurons against a variety of stressors, including ischemia-reperfusion [18], amyloid- $\beta$ [19], tau pathology [20], and aging [21]. Considerable data suggests that diminished Akt phosphorylation is associated with cognitive deficits in hyperglycemic conditions [22, 23]. pAkt can be reduced in hippocampus by hyperglycemia [23]. LCR rats display signs of type 2 diabetes with reduced whole body insulin sensitivity and hyperglycemia [16]. Our finding that pAkt is significantly lower in young adult LCR hippocampus is consistent with those previous findings. We previously reported lower mitochondrial markers (PGC1- $\alpha$, Nrf1 , and TFAM) in young adult LCR (6-7months) striatum, but not hippocampus [9]. Meanwhile, others have reported lower PGC1- $\alpha$ and TFAM in aged (23 months) LCR hippocampus, with concomitant cognitive deficits, hyperphosphorylated tau accumulation, and neuron loss [15]. These findings suggest that phosphorylation of Akt might be affected earlier than mitochondrial markers in LCR hippocampus. However, whether lower pAkt is accompanied by tau or amyloid- $\beta$ pathologies in young adult LCR hippocampus requires further investigation.

Phosphorylation of Akt is aberrantly regulated in hippocampus after HFD. One study reported clear 
$\mathbf{A}$
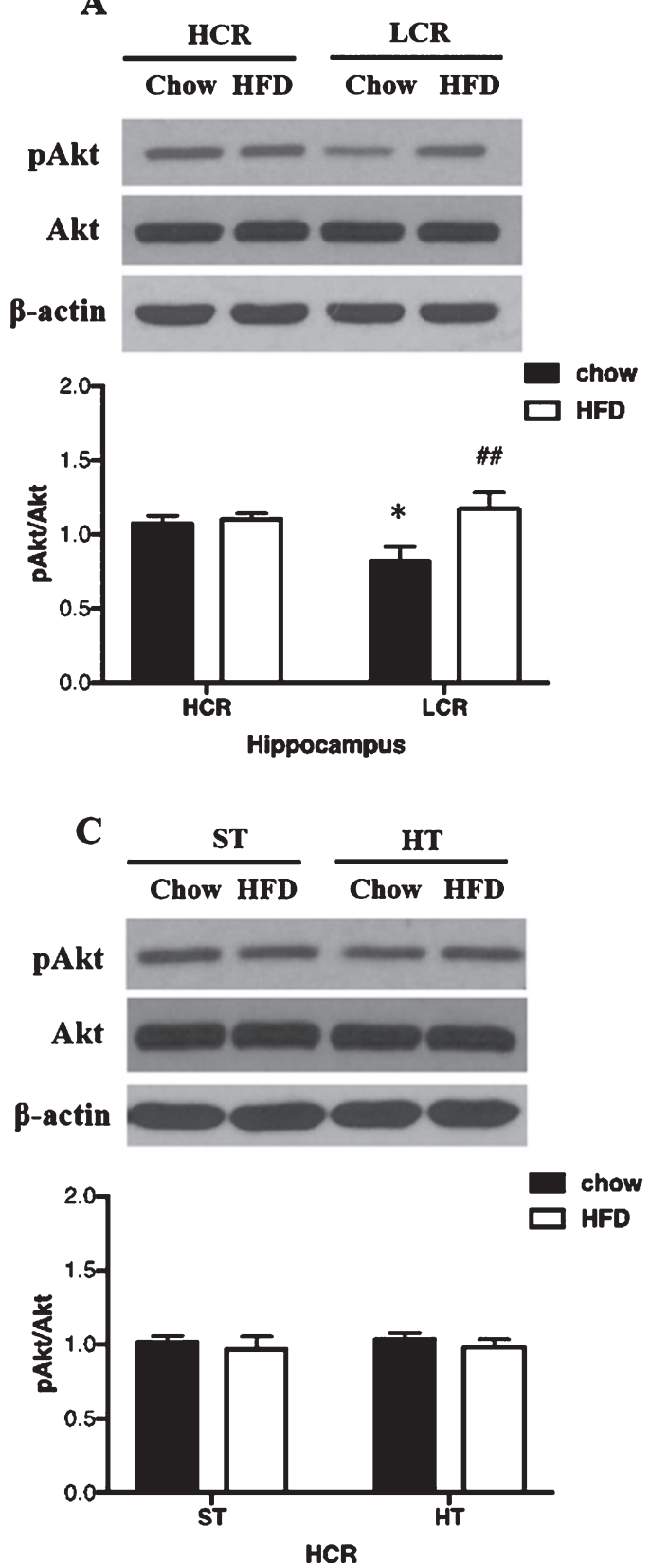

B

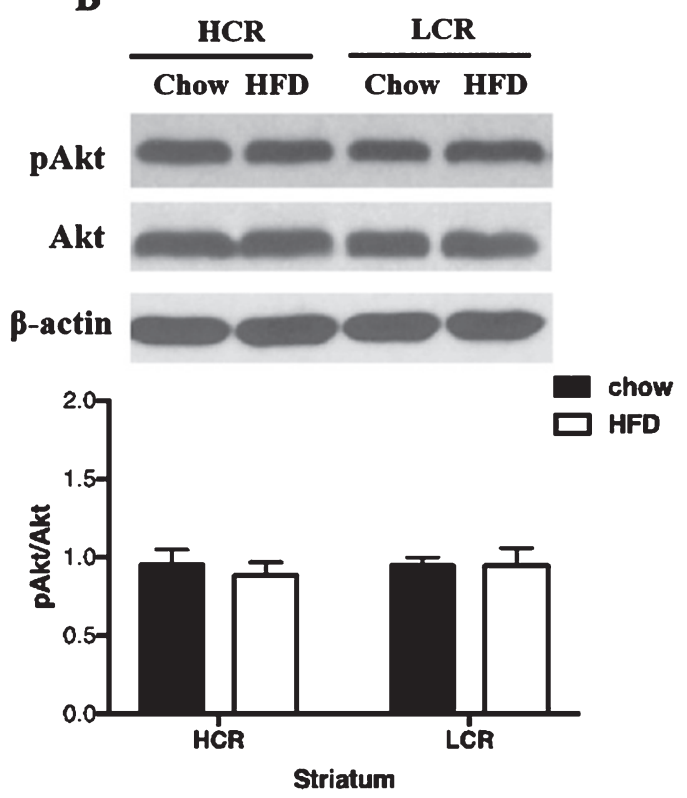

D
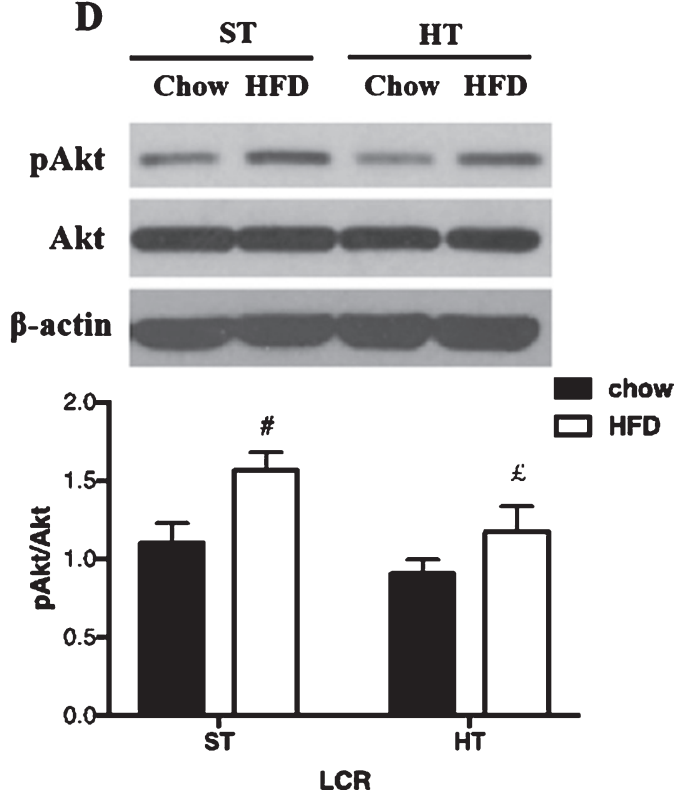

Fig. 1. A) Hippocampal and B) striatal pAkt/Akt expression in HCR/LCR with chow diet versus a 3-day HFD. pAkt/Akt expression in (C) HCR and (D) LCR rat hippocampus following HT. Rats were fed a chow diet or 3-day HFD and received either a single in vivo sham $\left(37^{\circ} \mathrm{C}\right)$ or heat $\left(41^{\circ} \mathrm{C}\right)$ treatment. Black bars represent chow diet and white bars represent 3-day HFD. Values are expressed as mean $\pm \mathrm{SE}$. $\mathrm{N}=5-6$ rats per group. ${ }^{*} p<0.05,{ }^{* *} p<0.01$ denotes significant difference from HCR versus LCR within diets (post hoc); ${ }^{\#} p<0.05,{ }^{\# \#} p<0.01$ denotes significant difference from chow diet versus HFD within strains (post hoc); ${ }^{£} p<0.05,{ }^{£ £} p<0.01$ denotes significant difference from HT versus sham treatment within diets (post hoc).

decreases in pAkt/Akt in rat hippocampus after a 10-week HFD [24]. Another study reported that a 5-month HFD resulted in elevated pAkt/Akt in rat hippocampus [25]. Here we provide evidence that despite whole body insulin resistance [16] and lower
pAkt in the LCR hippocampus with normal chow, LCR rats exhibited greater pAkt expression in hippocampus but no difference in pAkt in striatum after a 3-day HFD. LCR striatum has also exhibited greater mitochondrial proteins after a 3-day HFD [9]. Those 
A

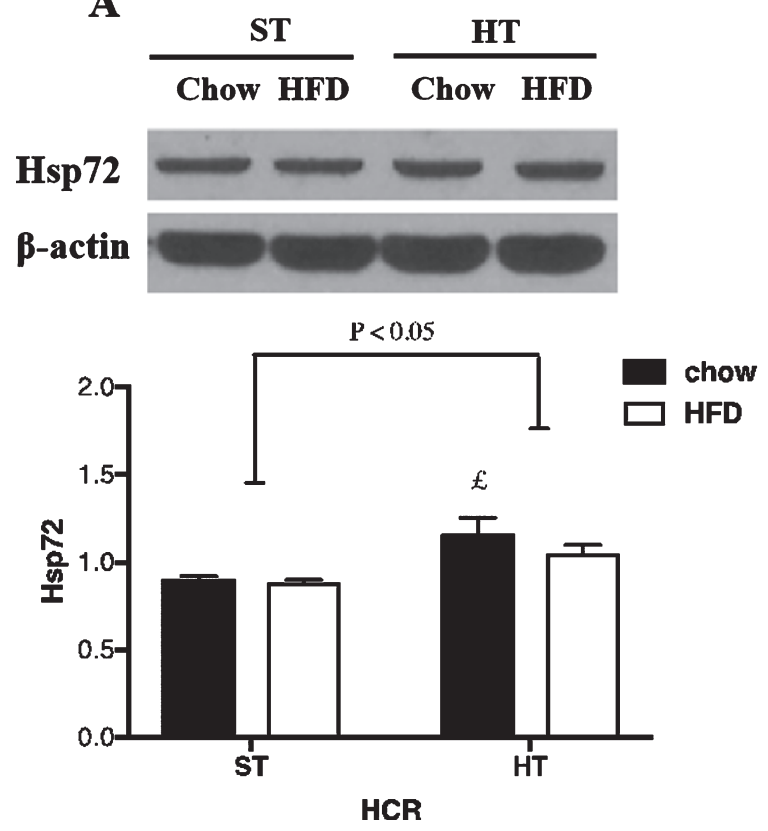

B

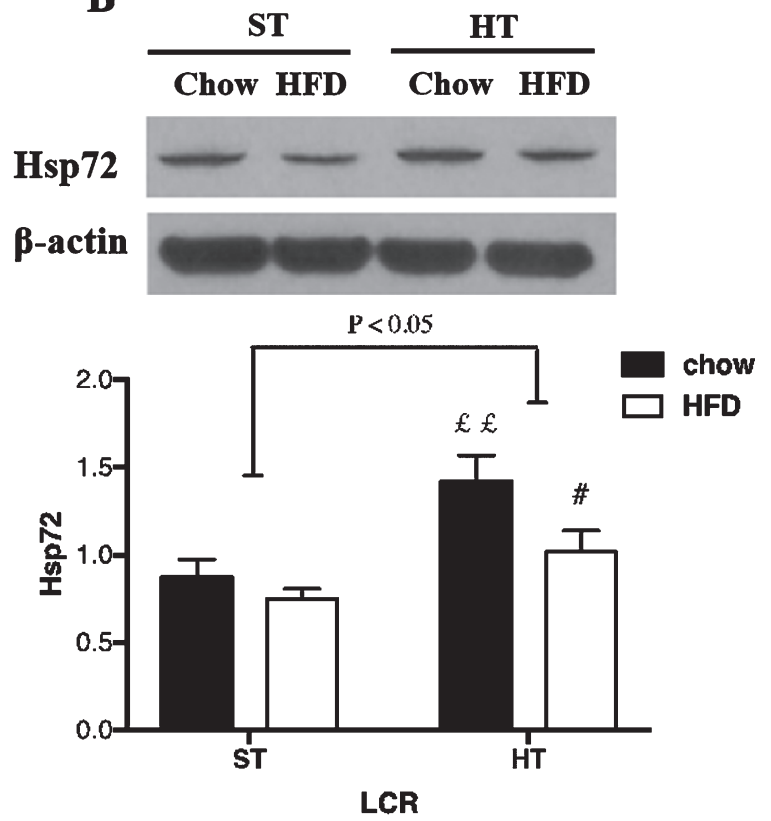

Fig. 2. HSP72 expression in (A) HCR and (B) LCR rat hippocampus following HT. Rats were fed a chow diet or 3-day HFD and received either a single in vivo sham $\left(37^{\circ} \mathrm{C}\right)$ or heat $\left(41^{\circ} \mathrm{C}\right)$ treatment. Black bars represent chow diet and white bars represent a 3-day HFD. Values are expressed as mean $\pm \mathrm{SE}$. $\mathrm{N}=5$-6 rats per group. ${ }^{\#} p<0.05,{ }^{\# \#} p<0.01$ denotes significant difference from chow diet versus HFD within treatments (post hoc). ${ }^{£} p<0.05,{ }^{£ £} p<0.01$ denotes significant difference from HT versus sham treatment within diets (post hoc).

data suggest the low aerobic capacity increases the vulnerability of brain to metabolic stress of HFD, and there are different responses to metabolic challenge in different brain regions.

HT can improve metabolic dysfunction in liver and skeletal muscles of rats followed HFD [16, 26]. Heat shock proteins (HSP) played a key role in protection following HT. One of the most studied HSPs in mammalian models is HSP72 (inducible HSP70), due to its protective effects in cerebral ischemia, neuronal cultures, cerebral trauma, and neurodegeneration [27-29]. HSP72 is a cytosolic chaperone, is highly inducible following heat stress, and has anti-oxidation, anti-inflammatory, and anti-apoptotic properties [30]. Our results show that HSP72 is elevated in both HCR and LCR rat hippocampus after HT. However, induction of HSP72 by HT is lower following a 3-day HFD than following a chow diet in LCR hippocampus. A previous study reported that LCR rats exhibit a blunted induction of HSP72 following HT in skeletal muscle, liver, and adipose tissue [16]. To our knowledge, our study is the first to report that low aerobic capacity is related to deficient induction of HSP72 following HT in hippocampus after a 3-day HFD. Meanwhile, we found that the elevating effects of a HFD on pAkt are reversed in LCR hippocampus after HT. Neither HFD nor heat stress affected pAkt in HCR hippocampus. Previous studies report that aged LCR rats exhibit worse spatial memory than HCR rats with eventual increases in hyperphosphorylated tau accumulation and neuron loss in hippocampus $[14,15]$. Our results suggest that the abnormal phosphorylation of Akt and diminished HSP response in the hippocampus of young adult LCR rats might indicate early vulnerability to metabolic challenges in this key brain region associated with learning and memory.

\section{ACKNOWLEDGMENTS}

This work was supported by Jiangxi Provincial Natural Science Foundation (20202BABL216024), the Science and technology plan of Jiangxi Health Committee (Grant NO. 20195207), the Youth Fund of The Second Affiliated Hospital of Nanchang University (Grant NO. 2019YNQN2011), NIH grants AG-031575, DK-088940, the KUMC Biomedical Research Training Program, and The Research and Training Project Funding of Nanchang University (ID: 4626). The LCR-HCR rat model system was funded by P40OD021331 (LGK and SLB). This 
model is maintained as an international resource with support from the Department of Physiology and Pharmacology at the University of Toledo, Toledo, Ohio (http://www.utoledo.edu/med/depts/physpharm/Exe rciseRatResources.html).

\section{CONFLICT OF INTEREST}

The authors have no conflict of interest to report.

\section{REFERENCES}

[1] Myers J, Prakash M, Froelicher V, Do D, Partington S, Atwood JE (2002) Exercise capacity and mortality among men referred for exercise testing. $N$ Engl J Med 346, 793801.

[2] Kodama S, Saito K, Tanaka S, Maki M, Yachi Y, Asumi M, Sugawara A, Totsuka K, Shimano H, Ohashi Y, Yamada N, Sone H (2009) Cardiorespiratory fitness as a quantitative predictor of all-cause mortality and cardiovascular events in healthy men and women: A meta-analysis. JAMA 301, 2024-2035.

[3] Kokkinos P, Myers J, Faselis C, Panagiotakos DB, Doumas M, Pittaras A, Manolis A, Kokkinos JP, Karasik P, Greenberg M, Papademetriou V, Fletcher R (2010) Exercise capacity and mortality in older men: A 20-year follow-up study. Circulation 122, 790-797.

[4] Church TS, Cheng YJ, Earnest CP, Barlow CE, Gibbons LW, Priest EL, Blair SN (2004) Exercise capacity and body composition as predictors of mortality among men with diabetes. Diabetes Care 27, 83-88.

[5] LaMonte MJ, Barlow CE, Jurca R, Kampert JB, Church TS, Blair SN (2005) Cardiorespiratory fitness is inversely associated with the incidence of metabolic syndrome: A prospective study of men and women. Circulation 112, 505512.

[6] Thyfault JP, Rector RS, Uptergrove GM, Borengasser SJ, Morris EM, Wei Y, Laye MJ, Burant CF, Qi NR, Ridenhour SE, Koch LG, Britton SL, Ibdah JA (2009) Rats selectively bred for low aerobic capacity have reduced hepatic mitochondrial oxidative capacity and susceptibility to hepatic steatosis and injury. J Physiol 587, 1805-1816.

[7] Wisløff U, Najjar SM, Ellingsen O, Haram PM, Swoap S, Al-Share Q, Fernström M, Rezaei K, Lee SJ, Koch LG, Britton SL (2005) Cardiovascular risk factors emerge after artificial selection for low aerobic capacity. Science 307, 418-420.

[8] Rivas DA, Lessard SJ, Saito M, Friedhuber AM, Koch LG, Britton SL, Yaspelkis BB, 3rd, Hawley JA (2011) Low intrinsic running capacity is associated with reduced skeletal muscle substrate oxidation and lower mitochondrial content in white skeletal muscle. Am J Physiol Regul Integr Comp Physiol 300, R835-843.

[9] Gan L, Ma D, Li M, Yang FC, Rogers RS, Wheatley JL, Koch LG, Britton SL, Thyfault JP, Geiger PC, Stanford JA (2018) Region-specific differences in bioenergetic proteins and protein response to acute high fat diet in brains of low and high capacity runner rats. Neurosci Lett 674, 49-53.

[10] Cole AR, Astell A, Green C, Sutherland C (2007) Molecular connexions between dementia and diabetes. Neurosci Biobehav Rev 31, 1046-1063.
[11] Strachan MW, Price JF, Frier BM (2008) Diabetes, cognitive impairment, and dementia. BMJ 336, 6.

[12] Feng X, Degos V, Koch LG, Britton SL, Zhu Y, Vacas S Terrando N, Nelson J, Su X, Maze M (2013) Surgery results in exaggerated and persistent cognitive decline in a rat model of the metabolic syndrome. Anesthesiology 118, 1098-1105.

[13] Wikgren J, Mertikas GG, Raussi P, Tirkkonen R, Äyräväinen L, Pelto-Huikko M, Koch LG, Britton SL, Kainulainen $\mathrm{H}$ (2012) Selective breeding for endurance running capacity affects cognitive but not motor learning in rats. Physiol Behav 106, 95-100.

[14] Sarga L, Hart N, Koch LG, Britton SL, Hajas G, Boldogh I, Ba X, Radak Z (2013) Aerobic endurance capacity affects spatial memory and SIRT1 is a potent modulator of 8oxoguanine repair. Neuroscience 252, 326-336.

[15] Choi J, Chandrasekaran K, Demarest TG, Kristian T, Xu S, Vijaykumar K, Dsouza KG, Qi NR, Yarowsky PJ, Gallipoli R, Koch LG, Fiskum GM, Britton SL, Russell JW (2014) Brain diabetic neurodegeneration segregates with low intrinsic aerobic capacity. Ann Clin Transl Neurol 1, 589-604.

[16] Robert S. Rogers EMM, Joshua L. Wheatley, Ashley E. Archer, Colin S., McCoin KSW, David R. Wilson, Grace M. E. Meers, Lauren G. Koch, Steven, L. Britton JPT, Paige C. Geiger (2016) Deficiency in the heat stress response could underlie susceptibility to metabolic disease. Diabetes 65, 3341-3351.

[17] Morris EM, Jackman MR, Johnson GC, Liu TW, Lopez JL, Kearney ML, Fletcher JA, Meers GM, Koch LG, Britton SL, Rector RS, Ibdah JA, MacLean PS, Thyfault JP (2014) Intrinsic aerobic capacity impacts susceptibility to acute high-fat diet-induced hepatic steatosis. Am J Physiol Endocrinol Metab 307, E355-364.

[18] Li P, Zhang Y, Liu H (2019) The role of Wnt/ $\beta$-catenin pathway in the protection process by dexmedetomidine against cerebral ischemia/reperfusion injury in rats. Life Sci $\mathbf{2 3 6}$, 116921.

[19] Martín D, Salinas M, López-Valdaliso R, Serrano E, Recuero M, Cuadrado A (2001) Effect of the Alzheimer amyloid fragment Abeta(25-35) on Akt:PKB kinase and survival of PC12 cells. J Neurochem 78, 1000-1008.

[20] Xu G-G, Deng Y-Q, Liu S-J, Li H-L, Wang J-Z (2005) Prolonged Alzheimer-like tau hyperphosphorylation induced by simultaneous inhibition of phosphoinositol-3 kinase and protein kinase C in N2a cells. Acta Biochim Biophys Sin 37, 349-354.

[21] Jackson TC, Rani A, Kumar A, Foster TC (2009) Regional hippocampal differences in AKT survival signaling across the lifespan: Implications for CA1 vulnerability with aging. Cell Death Differ 16, 439-448.

[22] Wang X, Zhao L (2016) Calycosin ameliorates diabetesinduced cognitive impairments in rats by reducing oxidative stress via the PI3K/Akt/GSK-3beta signaling pathway. Biochem Biophys Res Commun 473, 428-434.

[23] Kang S, Kim CH, Jung H, Kim E, Song HT, Lee JE (2017) Agmatine ameliorates type 2 diabetes induced-Alzheimer's disease-like alterations in high-fat diet-fed mice via reactivation of blunted insulin signalling. Neuropharmacology 113, 467-479.

[24] Yu H, Deng J, Zuo Z (2014) High-fat diet reduces neuroprotection of isoflurane post-treatment: Role of carboxyl-terminal modulator protein-Akt signaling. Obesity (Silver Spring) 22, 2396-2405.

[25] Muller AP, Cammarota M, Dietrich MO, Rotta LN, Portela LV, Souza DO, Izquierdo I, Bevilaqua LR, Perry ML (2008) 
Different effect of high fat diet and physical exercise in the hippocampal signaling. Neurochem Res 33, 880-885.

[26] Gupte AA, Bomhoff GL, Swerdlow RH, Geiger PC (2009) Heat treatment improves glucose tolerance and prevents skeletal muscle insulin resistance in rats fed a high-fat diet. Diabetes 58, 567-578.

[27] Paul S, Mahanta S (2014) Association of heat-shock proteins in various neurodegenerative disorders: Is it a master key to open the therapeutic door? Mol Cell Biochem 386, 45-61.
[28] Kim JY, Han Y, Lee JE, Yenari MA (2018) The 70-kDa heat shock protein (Hsp70) as a therapeutic target for stroke. Expert Opin Ther Targets 22, 191-199.

[29] Kim N, Kim JY, Yenari MA (2015) Pharmacological induction of the $70-\mathrm{kDa}$ heat shock protein protects against brain injury. Neuroscience 284, 912-919.

[30] Rogers RS, Beaudoin MS, Wheatley JL, Wright DC, Geiger PC (2015) Heat shock proteins: in vivo heat treatments reveal adipose tissue depot-specific effects. J Appl Physiol (1985) 118, 98-106. 